\title{
Analysis of the Electronic Structure of Aqueous Urea and its Derivatives: A Systematic Soft X-Ray - TD-DFT Approach
}

\author{
Marc F. Tesch, ${ }^{* a]}$ Ronny Golnak, ${ }^{[a, b]}$ Felix Ehrhard,,${ }^{[a]}$ Daniela Schön,,${ }^{[a, ~ c]}$ Jie Xiao, ${ }^{[a]}$ Kaan Atak, ${ }^{[a]}$ \\ Annika Bande, ${ }^{[a]}$ and Emad F. Aziz ${ }^{*[a, c]}$
}

\begin{abstract}
Soft X-ray emission, absorption, and resonant inelastic scattering experiments have been conducted at the nitrogen $\mathrm{K}$-edge of urea and its derivatives in aqueous solutions and compared with density functional theory and time-dependent density functional theory calculations. This comprehensive study provides detailed information on the occupied and unoccupied molecular orbitals of urea, thiourea, acetamide, dimethylurea and biuret at valence levels. By identifying the electronic transitions that contribute to the experimental spectral features the energy gap between the highest occupied and the lowest unoccupied molecular orbital of each molecule is determined. Moreover, a theoretical approach is introduced to simulate resonant inelastic X-ray scattering spectra by adding an extra electron to the lowest unoccupied molecular orbital mimicking the real initial state of the core electron absorption before the subsequent relaxation process.
\end{abstract}

\section{Introduction}

Obtaining a comprehensive knowledge about the electronic structure of solvated molecules is a major task for the understanding of chemical reactions and physical properties of a molecule. To achieve this goal, a straightforward approach is the application of synchrotron radiation based spectroscopies, since they allow for an investigation of the electronic structure at an element selective level by tuning the photon energy to the absorption edges of the elements of interest. The application of such techniques on liquids, however, remains a challenging task, since the use of soft $X$-rays needs a vacuum environment.

[a] Dr. M. F. Tesch*, R. Golnak, F. Ehrhard, D. Schön, Dr. J. Xiao Dr. K. Atak, Dr. A. Bande, Prof. E. F. Aziz* Institute of Methods for Material Development Helmholtz-Zentrum Berlin für Materialien und Energie Albert-Einstein-Straße 15, 12489 Berlin, Germany *E-mail: emad.aziz@helmholtz-berlin.de

b] R. Golnak marc.tesch@helmholtz-berlin.de

Department of Chemistry

Freie Universität Berlin

Takustraße 3, 14195 Berlin, Germany

[c] D. Schön, Prof. E. F. Aziz

Department of Physics

Freie Universität Berlin

Arnimallee 14, 14195 Berlin, Germany
Nevertheless, significant achievements have been made due to the ongoing development of flow-cell and liquid micro-jet techniques ${ }^{[1-6]}$ in recent years.

This study focuses on the electronic structure of aqueous urea. Although urea is widely studied (see e.g. references 7-15) an examination of its electronic structure in liquid environment is still lacking, until now. The knowledge about the electronic structure of urea in solution, however, is crucial for a deeper understanding of its behavior in chemical reactions. In particular, the energy gap between the highest occupied molecular orbital (HOMO) and the lowest unoccupied molecular orbital (LUMO) is of large interest since it serves as indicator for molecular properties like stability or reactivity (see, e.g., references 16-19 and references therein). The HOMO-LUMO gap itself, however, is strongly affected by an exchange of the functional groups of a molecule. To examine this influence, a systematic investigation on the urea derivatives thiourea, acetamide, dimethylurea, and biuret (Figure 1) was conducted as well.

X-ray absorption spectroscopy (XAS), X-ray emission spectroscopy (XES), and resonant inelastic X-ray scattering (RIXS) were performed at the nitrogen K-edges of the molecules. These are powerful techniques, enabling an investigation of the occupied and unoccupied electronic states as well as valence excitations. ${ }^{[20]}$ The experimental emission and absorption spectra are compared to density functional theory (DFT) calculations and time-dependent density functional theory (TD-DFT) calculations, respectively. This allows to identify the orbital contributions within the spectra, which is important for a reliable determination of the HOMO-LUMO gap from the experimental data.

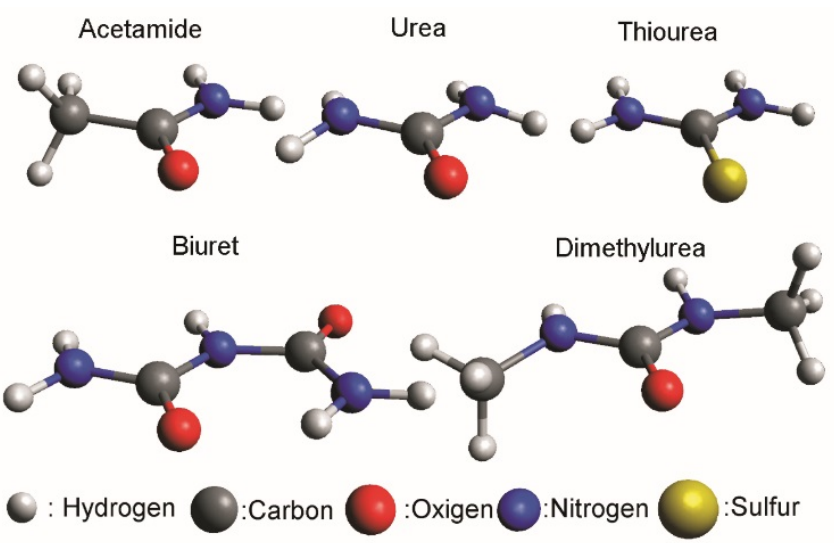

Figure 1. Schematics of the molecular geometric structures of urea, and its derivatives acetamide, thiourea, dimethylurea, and biuret. 


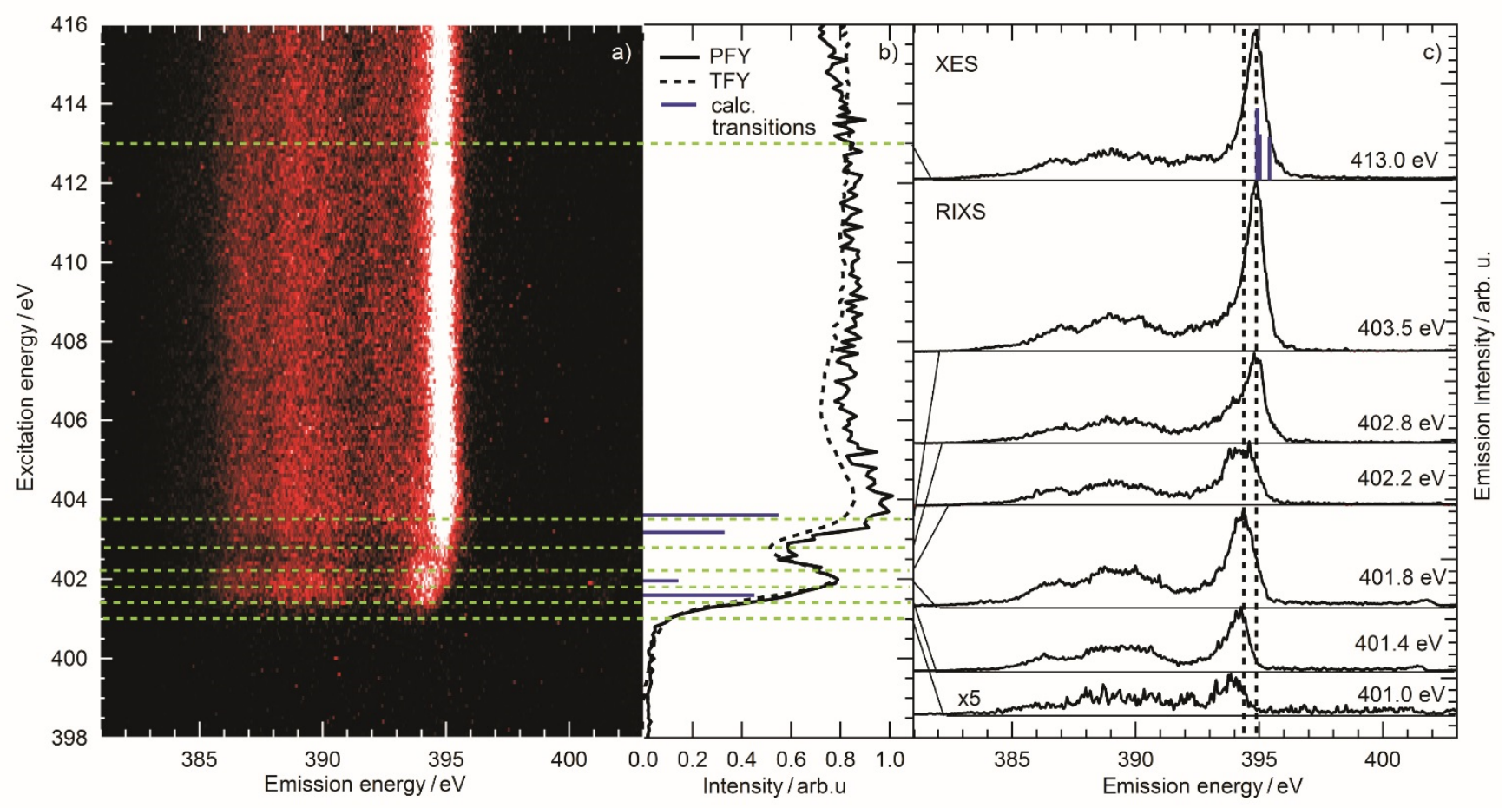

Figure 2. a) Spectral image of the energy resolved X-ray emission at different excitation energies and b) X-ray absorption spectra for urea measured in partial and total fluorescence yields. Part c) shows X-ray emission spectra at several selected excitation energies (green lines in middle panel a) and b) ). The blue bars indicate the calculated transition moments.

A new approach is presented to simulate the X-ray emission process with different initial states, one is the commonly-adopted neutral ground state and the other is the newly introduced negatively-ionized state with one extra electron occupying the former LUMO. It will show that including the influence of an additional electron in the calculations can reproduce the experimental shifts within the RIXS process.

In section II.1 the electronic structure of urea will be investigated by a detailed analysis of the combined XA/XE and RIXS spectra that are further compared to the theoretical calculations. A brief analysis of the urea derivatives thiourea, acetamide and dimethylurea will be given in section II.2. The discussion of the biuret molecule, another urea derivative with two carboxamide groups, appears as a special case in section II.3.

\section{Results and Discussion}

The HOMO-LUMO gaps of the five investigated molecules are determined by a comparison of the experimental XA and XE spectra to the computed ones, including the analysis of the contributions of the computed MOs to the spectral features. Although the excitation energies are underestimated by the calculations, ${ }^{[21]}$ the calculated relative energies between the electronic transitions still hold physical significance, and therefore are used for comparison with the experimental spectra. The calculated spectra were shifted by the same amount of $20.2 \mathrm{eV}$ to higher energies to allow a direct comparison of the calculated spectra among each other. The good accordance to the relative energetic shifts in the line-up of the five molecules demonstrates the accuracy of the used theoretical approach.

\section{II.1 Urea}

The experimental spectra from $1 \mathrm{M}$ aqueous urea solution are shown in Figure 2. Several studies have demonstrated that for this concentration an urea-urea interaction, which might lead to additional features in the spectra, is very unlikely ${ }^{[11,22,23]}$ and therefore is neglected in the further discussion. In the left panel the energy resolved X-ray emission of the urea molecule is shown for an incident photon energy tuned from 398.0 to $416.0 \mathrm{eV}$ across the nitrogen K-edge in steps of $0.1 \mathrm{eV}$. The amount of photons emitted by the sample, measured in total fluorescence yield (TFY), as well as in partial fluorescence yield (PFY) (Figure 2 b) ), is assumed to be proportional to the absorption coefficient of the molecule. ${ }^{[24]}$ The absorption spectra exhibit two features, one sharp peak at $402.0 \mathrm{eV}$ and one broader peak around $404.0 \mathrm{eV}$, which will be analyzed in detail.

The comparison of the experimental absorption spectra to the calculated transition moments unravels the details about the nature of the contributing orbitals. The two lowest unoccupied orbitals (blue bars at $401.63 \mathrm{eV}$ and $402.01 \mathrm{eV}$ in Figure $2 \mathrm{~b}$ )) contribute to the first experimental peak. The LUMO exhibits a $\pi^{*}$ character with respect to the $\mathrm{C}-\mathrm{N}$ and $\mathrm{C}-\mathrm{O}$ bonds, but has a more binding $\pi$-like character for the $\mathrm{N}-\mathrm{H}$ bonds (see Figure 3 ). The LUMO +1 also has a $\pi^{*}$ character for these bonds but a predominantly $\sigma^{*}$ character at the $\mathrm{N}-\mathrm{H}$ bonds. The $\mathrm{N} 1 \mathrm{~s} \rightarrow$ LUMO transition, the first blue bar at $401.63 \mathrm{eV}$, contributes to $\sim 75 \%$ to the first peak and the $\mathrm{N} 1 \mathrm{~s} \rightarrow \mathrm{LUMO}+1$ transition, the second blue 
bar at $402.01 \mathrm{eV}$, contributes to $\sim 25 \%$ (see Figure $2 \mathrm{~b}$ ) ). Both transitions are energetically separated by $0.38 \mathrm{eV}$ (Figure 3 ) and therefore appear as one single peak in the experiment. The next two energetically higher transitions, marked by two blue bars at 403.26 and $403.59 \mathrm{eV}$, exhibit an energy splitting of $0.33 \mathrm{eV}$ and can be assigned to the broad peak at $404.0 \mathrm{eV}$. Both transitions contain the LUMO+2 and the LUMO+3.

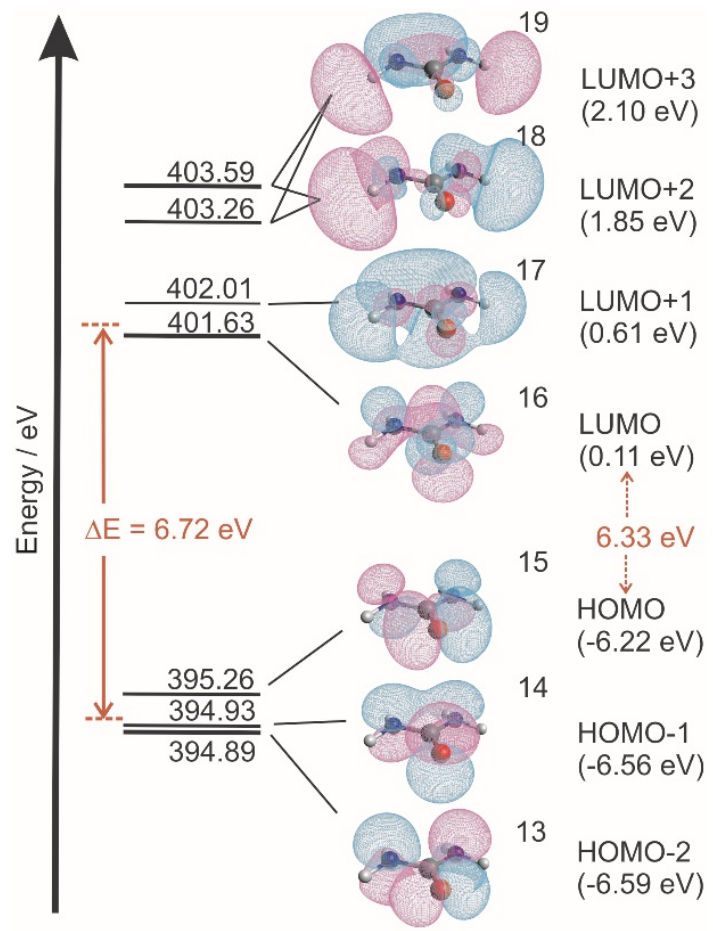

Figure 3. Calculated energies of the absorption and emission transitions and the corresponding orbital shapes and energies of the three highest occupied and four lowest unoccupied molecular orbitals of urea. The energy gap $\Delta \mathrm{E}=6.72$ $\mathrm{eV}$ is read from the peak positions of the simulated emission and absorption spectra (cf. Figure 4). The ordinal numbers besides the visualized orbitals correspond to the numbers in Figure 4

The X-ray emission spectrum (Figure $2 \mathrm{c}$ ), top) was measured at $413.0 \mathrm{eV}$ excitation energy, i.e., it is the relaxation of a valence electron to the $\mathrm{N}$ 1s core hole originating from an off-resonant excitation. It exhibits one prominent peak at $394.85 \mathrm{eV}$ and several smeared out features at lower energies. A closer look at the theoretical calculations reveals that three transitions (indicated by the blue bars in Figures 2 and 4 a) ) contribute to the peak at $394.85 \mathrm{eV}$. These stem from the HOMO, HOMO-1, and HOMO-2. The numbering in Figure 4 a) and Figure 3 assigns the transitions to the contributing orbitals. The $\mathrm{HOMO} \rightarrow \mathrm{N} 1 \mathrm{~s}$ and the HOMO-1 $\rightarrow \mathrm{N}$ 1s transition, indicated in Figure $2 \mathrm{c}$ ) by the blue bars at $395.26 \mathrm{eV}$ and $394.93 \mathrm{eV}$, respectively, contribute equally to the emission spectrum, while the intensity of the HOMO-2 $\rightarrow$ N 1s transition (blue bar at $394.89 \mathrm{eV}$ ) is almost $50 \%$ higher than the intensity of the other two transitions. This difference originates in the respective contributions of the different atoms to the MOs involved in the relaxation process (Figure 3). While the contribution of the oxygen atom to the HOMO and the HOMO- 1 is calculated to be approximately $50 \%$, the two nitrogen atoms have an overall contribution of only $~ 35 \%$ to these orbitals. For the HOMO-2, on the other hand, the contribution of the two nitrogen sites exceeds $60 \%$, while the oxygen contributes to $23 \%$. Since the core hole is located at one nitrogen site of the molecule, the recombination probability of an electron from the HOMO-2, (exhibiting a large nitrogen contribution) to a $\mathrm{N} 1 \mathrm{~s}$ core hole is higher than that for an electron from the HOMO and HOMO-1, (both exhibiting a lower nitrogen contribution). The central carbon atom is barely contributing to all of the three orbitals and hence the $\mathrm{C}-\mathrm{O}$ and $\mathrm{C}-\mathrm{N}$ bonds appear to have mainly non-bonding character (Figure 3 ).

A Gaussian broadening of $1 \mathrm{eV}$ is applied to each calculated transition moment (blue bar) to simulate the experimental spectra, as shown in Figure 4, where the simulated spectra is shown as red curves. The height of the bars represents the relative transition probability. The maximum intensity of each spectrum in Figure 4 is normalized to unity, while the height of the maximum transition in the line spectra are normalized to 0.5 . The left part of the spectrum in Figure 4 a) illustrates the emission for the ionized urea molecule (cf. Figure $2 \mathrm{c}$ ) ) and the right part of Figure $4 \mathrm{a}$ ) shows the absorption spectrum, measured in PFY (cf. Figure 2 b) ). For the simulated spectrum (red lines in Figure 4 a) ) the above discussed transitions merge into single features resulting in a main feature in the XE spectrum and one main feature in the $\mathrm{XA}$ spectrum at $394.99 \mathrm{eV}$ and $401.67 \mathrm{eV}$, respectively. The energy gap $\Delta E$ between the calculated features is in excellent agreement with the experimental peak-to-peak value. In the energy region below $393.0 \mathrm{eV}$ the experimental spectrum exhibits smeared-out features in contrast to the clearly distinguishable features predicted by the calculation. This discrepancy between experiment and theory might originate from the interactions of urea with solvent water which is lacking in the single molecule calculations. The MOs at this energy region form mostly $\sigma$-like bonds. The energetic position of a $\sigma$ orbital, however, is sensitive to changes of the bond length. ${ }^{[20]}$ The interaction with neighboring molecules can result in a slight variation of the $\sigma$ bond length. Therefore, for a liquid - implying a large amount of molecular interactions resulting in different molecular configurations - these features might appear to be smeared out. The calculated spectrum based on an averaging field created by the solvent, however, does not consider such effects.

The discrepancies between the experimental and calculated spectra in the high energy region above $\sim 404.0 \mathrm{eV}$ originates from the continuum states of the molecule, which are neglected in the calculated spectrum. The continuum states above the ionization potential undergo a larger broadening than the localized ones. This deviations in the high energetic part of the absorption spectra and the low energetic part of the emission spectra, however, does not affect the further considerations since this study focuses on the positions of the highest occupied and lowest unoccupied MOs, which can be well reproduced by the calculations. 


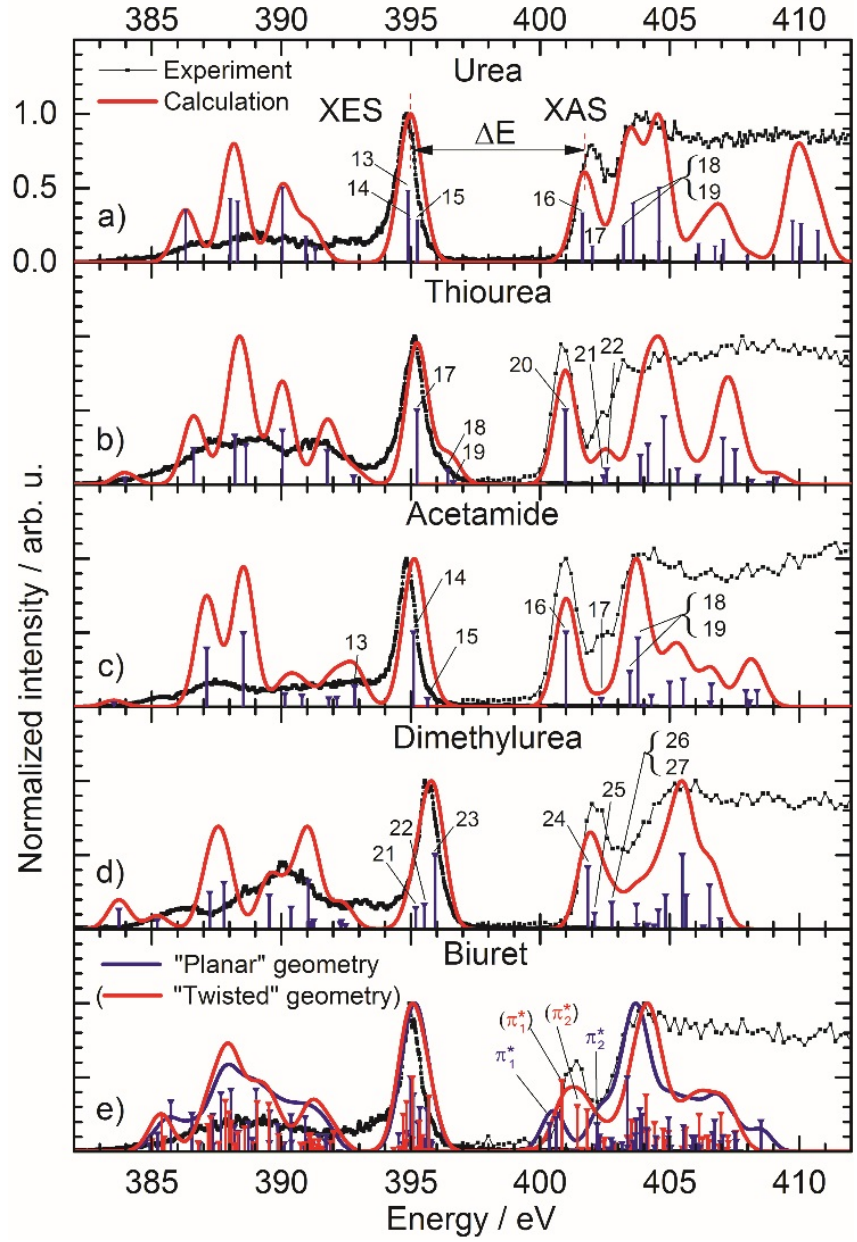

Figure 4. Experimental (black) and calculated (red) XE and XA spectra of the five investigated molecules. The vertical bars represent the calculated transition moments. The numbers indicate the contributing molecular orbitals (cf. Figures 3, 6 and 8). All XES spectra were taken at least $4 \mathrm{eV}$ above the IP.

A simple approach to estimate the HOMO-LUMO gap from the experiment is to look at the difference between the energetically lowest feature in the XA spectrum and the energetically highest feature in the XE spectrum under the assumption that neither the $\mathrm{N} 1 \mathrm{~s} \rightarrow$ LUMO transition nor the HOMO $\rightarrow \mathrm{N}$ 1s transition is dipole forbidden. However, one has to be careful when applying this simple model. From the calculations one can see in the combined XE/XA spectra of urea (Figure 4 a) ) that the XE-peak-to-XA-peak gap of the calculated spectrum is $\Delta E_{\text {calc }}^{p p}=6.72 \mathrm{eV}$, while the actual calculated HOMO-LUMO gap is $\Delta E_{\text {calc }}^{H L}=6.33 \mathrm{eV}$ (cf. Figure 3), i.e. the peak-to-peak difference overestimates the HOMO-LUMO gap by $0.39 \mathrm{eV}$. This deviation has to be taken into account when estimating the HOMO-LUMO gap from the experiment. The experimental spectra exhibit a peak-to-peak energy difference of $\Delta E_{\text {exp }}^{p p}=7.15 \mathrm{eV}$. When assuming a similar distribution of the transition moments underlying the experimental peaks as for the calculation the peak-to-peak value has to be corrected by subtracting the calculated deviation of $0.39 \mathrm{eV}$ to obtain a reliable experimental value of the HOMO-LUMO gap of $\Delta E_{\text {exp }}^{H L} \approx 6.75 \mathrm{eV}$ (cf. Table 1). This value is significantly larger than the band gap value of $6.2 \mathrm{eV}$ measured for crystalline urea, ${ }^{[25]}$ demonstrating the trend of the energy gap opening when going from a crystal structure with strong intermolecular interaction to the "isolated" urea molecule.

A proper interpretation of the RIXS spectra is more complicated due to intramolecular reorganization of the electronic structure when the excited electron occupies a formerly unoccupied orbital. The difference to the XE spectrum can be clearly seen in Figure 2 a). For seven selected incident photon energies detailed RIXS spectra are shown in Figure $2 \mathrm{c}$ ). The vertical dashed lines emphasize the energetic shift of the spectral features.

Two energy regions above the absorption edge can be identified. The first energy region is between 401.0 and $403.0 \mathrm{eV}$ where the LUMO and the LUMO+1 are occupied. The main emission feature at $\sim 394.5 \mathrm{eV}$ is strongest at an excitation energy of $\sim 401.8 \mathrm{eV}$. The emission main peak shifts slightly to higher energies when tuning the excitation energy from the pre-edge region to the absorption peak at $402.0 \mathrm{eV}$. In this first energy region beside the main peak an additional feature is visible as a shoulder at 394.0 $\mathrm{eV}$ to the main peak. This shoulder is not reproduced by the calculated transitions. Comparing the RIXS and the XE spectra this shoulder leads to a broadening of the RIXS main peak by $\sim 45 \%$, i.e. the full width at half maximum (FWHM) of the peak is $\sim 1.30 \mathrm{eV}$ in the RIXS spectra compared to $\sim 0.90 \mathrm{eV}$ in the XE spectra. In the study of Weinhardt et al. ${ }^{[26]}$ where N K-edge RIXS measurements were performed on ammonia a similar shoulder is associated to an ultrafast hydrogen dissociation process when the excited $\mathrm{N}$ 1s electron occupies the LUMO. This is discussed in detail at the end of section II.2. The second energy region above $403.0 \mathrm{eV}$ contains the excitations to the LUMO+2 and higher orbitals. Here, the overall spectrum is almost independent of the excitation energy and the RIXS spectra converges to the XE spectrum.

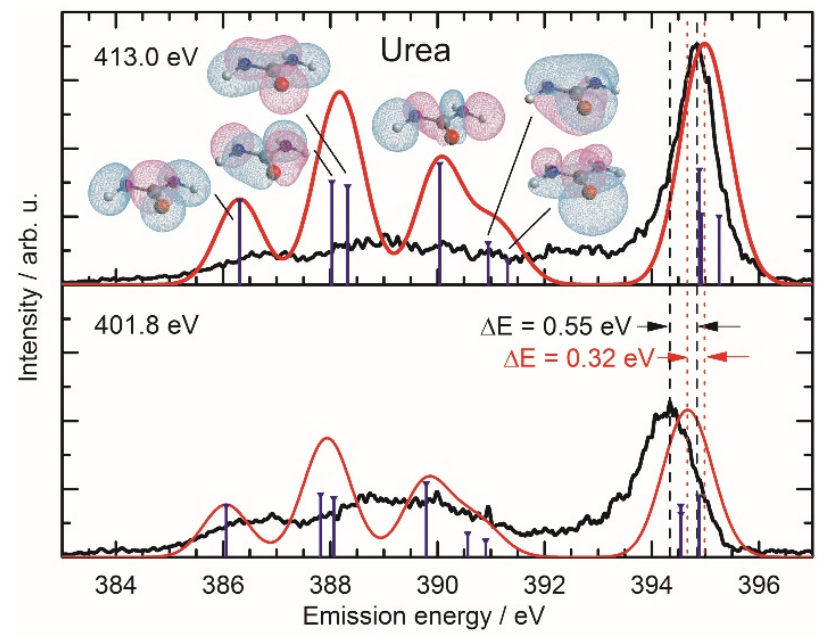

Figure 5. Experimental (black) and calculated (red) X-ray emission spectrum for the ionized urea molecule at the excitation energy of $413.0 \mathrm{eV}$ (top) and resonant inelastic X-ray scattering spectrum (black) and its simulated theoretical spectrum (red) at the excitation energy of $401.8 \mathrm{eV}$ (bottom). The dashed vertical lines emphasize the shifts of the main features. 
Taking into account the rearrangement of the electronic configuration during a RIXS process, the electronic transitions for the corresponding XE spectrum were calculated for an urea anion, i.e., the urea molecule was electronically relaxed with one additional electron occupying the former LUMO. The geometries of the neutral and charged molecules were kept the same in the calculations, i.e. it was assumed that the electronic reorientation and the recombination process determined by the core-hole lifetime of a few femtoseconds ${ }^{[27]}$ is faster than the structural reorganization of the molecule. The calculated spectrum (red line in Figure 5, bottom panel) clearly reproduces the experimentally observed shift of the main peak. A comparison of the underlying transitions for the spectra at excitation energies of $413.0 \mathrm{eV}$ and $401.8 \mathrm{eV}$ reveals that the spectral changes are caused by two effects. The first effect is the shift of the energy positions of the transition moments to lower energies. The former $\mathrm{HOMO} \rightarrow \mathrm{N}$ 1s transition, e.g., is shifted by $0.37 \mathrm{eV}$ to $394.98 \mathrm{eV}$. The second effect is the alteration of the transition probabilities, indicated by the height variations of the vertical blue bars for the simulated RIXS process in Figure 5. For the excited molecule the transition from the former HOMO to the $\mathrm{N}$ 1s core hole is stronger than the same transitions in the XE spectrum of the ionized molecule (Figure 5, top). This leads to an additional shift of the main peak back to higher energies. Both effects result in a calculated overall shift of $0.32 \mathrm{eV}$ to lower energies, compared to $0.55 \mathrm{eV}$ in the experiment. This deviation, however, may be due to the simple ansatz of only taking the extra valence electron into account but neglecting the core hole when approximating the RIXS spectrum calculations.

As an intermediate result it can be stated that the experimental $X A$ and XE spectra can be well described by TD-DFT calculations and the trend of the spectral changes for the RIXS spectra can be retraced when performing the same calculations for an urea anion. The deviations to the experiment, especially in the low energy part of the XES, might be caused by the neglected hydrogen bonding and altered intramolecular bond length caused by moleculesolvent interaction. The chemically more relevant orbitals, i.e. the HOMO and LUMO and the neighboring orbitals, however, can be well reproduced. Moreover, the good agreement of the experiment with the calculations that use a geometric optimized structure for urea in an implicit continuum water model shows that the surrounding water molecules seem to have a minor effect on the electronic structure of urea.

\section{II.2 Thiourea, Acetamide, and Dimethylurea}

In the following section the three urea derivatives thiourea, acetamide, and dimethylurea will be discussed. All three derivatives are of similar size and geometry as the urea molecule. The discussion will be brief and follows the same proceeding as for the urea molecule, i.e. the XE and XA spectra of the three molecules will be analyzed first and the HOMO-LUMO gap will be determined. The RIXS spectra of all three molecules will be examined at the end of this section.

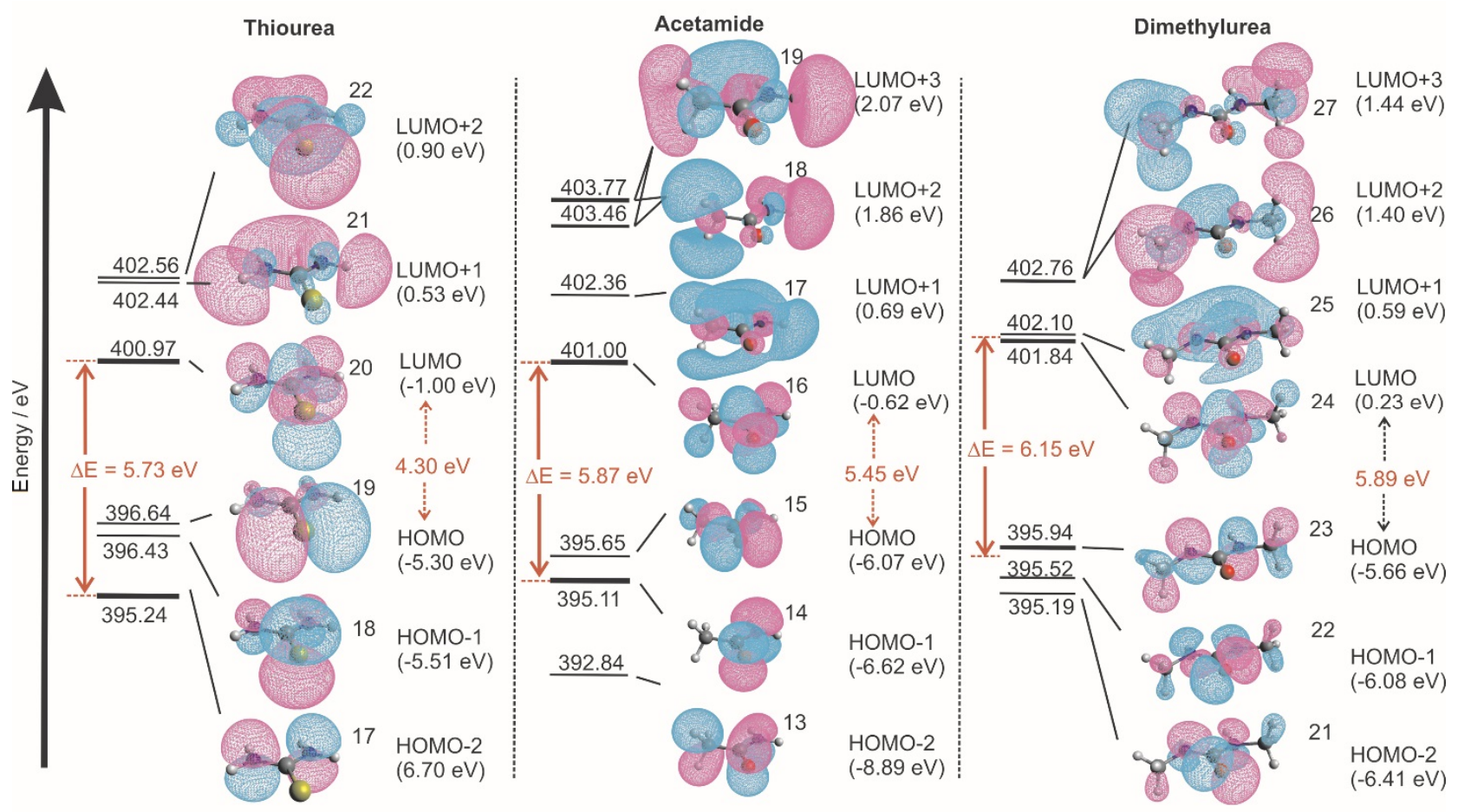

Figure 6 Calculated transition and orbital energies and spatial distribution of the highest occupied and lowest unoccupied molecular orbitals of thiourea, acetamide, and dimethylurea and the resulting transition and HOMO-LUMO gaps. $\Delta \mathrm{E}$ indicates the calculated peak-to-peak values of the simulated spectra (cf. Figure 4 ). The ordinal numbers besides the visualized orbitals correspond to the numbers in Figure 4. 
Table 1: Energy positions of the experimental and calculated features, the calculated HOMO-LUMO gap $\Delta \boldsymbol{E}_{\text {calc }}^{H L}$ and the estimated experimental HOMO-LUMO gap $\Delta \boldsymbol{E}_{\text {exp }}^{H L}$ (for details see text).

\begin{tabular}{|c|c|c|c|c|c|c|c|c|}
\hline & \multicolumn{2}{|c|}{$\begin{array}{l}\text { Experimental } \\
\text { peak-position }(\mathrm{eV})\end{array}$} & \multirow{2}{*}{$\begin{array}{l}\Delta E_{\text {exp }}^{p p}(\mathrm{eV}) \\
( \pm 0.10 \mathrm{eV})\end{array}$} & \multicolumn{2}{|c|}{$\begin{array}{l}\text { Calculated } \\
\text { peak-position }(\mathrm{eV})\end{array}$} & \multirow{2}{*}{$\Delta E_{c a l c}^{p p}(\mathrm{eV})$} & \multirow{2}{*}{$\Delta E_{\text {calc }}^{H L}(\mathrm{eV})$} & \multirow{2}{*}{$\begin{array}{l}\Delta \boldsymbol{E}_{\text {exp }}^{H L}(\mathrm{eV}) \\
( \pm 0.10 \mathrm{eV})\end{array}$} \\
\hline & $\begin{array}{l}\text { XES } \\
( \pm 0.05)\end{array}$ & $\begin{array}{l}\text { XAS } \\
( \pm 0.05)\end{array}$ & & XES & XAS & & & \\
\hline Urea & 394.85 & 402.00 & 7.15 & 394.98 & 401.70 & 6.72 & 6.33 & 6.75 \\
\hline Thiourea & 395.15 & 400.85 & 5.70 & 395.24 & 400.97 & 5.73 & 4.30 & 4.30 \\
\hline Acetamide & 394.85 & 400.95 & 6.10 & 395.13 & 401.00 & 5.87 & 5.45 & 5.70 \\
\hline Dimethylurea & 395.65 & 402.10 & 6.45 & 395.79 & 401.94 & 6.15 & 5.89 & 6.20 \\
\hline
\end{tabular}

Thiourea is the higher chalcogene homologue of urea, having the oxygen atom exchanged by a sulfur atom (Figure 1). The absorption spectrum of thiourea (right part of Figure 4 b) ) exhibits one well distinguishable peak located at $400.85 \mathrm{eV}$, which is energetically lower compared to the first absorption peak of Urea. This energetic shift can be well reproduced by the calculations. The fist absorption peak of thiourea at $400.85 \mathrm{eV}$ can be identified by the calculations as a single transition to the LUMO which has $\pi^{*}$ character for the C-N and C-S bond and $\pi$-character for the $\mathrm{N}-\mathrm{H}$ bonds. The $\sigma^{*}$-like LUMO+1 has an antibonding character for the $\mathrm{N}-\mathrm{H}$ and $\mathrm{C}-\mathrm{N}$ bonds and bonding character for the $\mathrm{C}-\mathrm{S}$ bond, whereas the LUMO+2 has an antibonding $\sigma^{*}$-character for the $\mathrm{C}-\mathrm{S}$ and the $\mathrm{N}-\mathrm{H}$ bonds and a bonding $\pi$ character for the $\mathrm{C}-\mathrm{N}$ bond. The transitions to these orbitals occur in the experiment as a shoulder at approximately $402.5 \mathrm{eV}$. The steep increase around $403.0 \mathrm{eV}$ is related to the transitions to the continuum states beyond the IP of thiourea.

The XE spectrum (Figure $4 \mathrm{~b}$ ) ) exhibits one prominent feature located at $395.15 \mathrm{eV}$, which, by comparison to the calculated line spectrum can be assigned to the HOMO-2 $\rightarrow \mathrm{N}$ 1s transition. An inspection on the MOs in Figure 6 reveals that the HOMO-2 is clearly dominated by the $\mathrm{N}$ sites of the molecule with a contribution of nearly $90 \%$, leading to a high transition probability from the HOMO-2 to the $\mathrm{N}$ 1s core hole. The HOMO and the HOMO-1, on the other hand, are located almost completely at the $S$ site of the molecule with a sulfur-contribution to the orbitals of $\sim 87 \%$ and $\sim 74 \%$, respectively. Therefore, these orbitals barely contribute to the relaxation process that involves a $\mathrm{N}$ core hole. The corresponding features in the emission spectrum only appear as a very small shoulder at $\sim 396.5 \mathrm{eV}$. Like in the case of urea the three highest occupied orbitals exhibit nearly no contribution of the central $\mathrm{C}$ atom and therefore are assigned to have a predominately non-bonding character regarding the C-S and C-N bonds, as is depicted in Figure 6.

The experimental HOMO-LUMO gap (Table 1) can be established based on the orbital information provided above. The experimental peak-to-peak value is $\Delta E_{\text {exp }}^{p p}=5.7 \mathrm{eV}$ thiourea. This value, however, has to be handled with care, since it was shown that the XE main peak is caused by transitions to the HOMO-2. The peak-to-peak value of the broadened calculated spectrum is $\Delta E_{\text {calc }}^{p p}=5.73 \mathrm{eV}$, which is in excellent agreement with the experiment. The corresponding calculated HOMO-LUMO gap is
$\Delta E_{\text {calc }}^{H L}=4.30 \mathrm{eV}$. This strong deviation to the peak-to-peak value needs to be taken into account for the experimental spectra, leading to an estimated HOMO-LUMO gap of also $\Delta E_{\text {exp }}^{H L} \approx 4.30 \mathrm{eV}$. A similar value of $4.35 \mathrm{eV}$ for the HOMO-LUMO gap was also obtained by UV/VIS experiments. ${ }^{[28]}$

Similar to the XA spectrum of thiourea the first XA peak for acetamide (Figure $4 \mathrm{c}$ ) ) at $400.95 \mathrm{eV}$ can be related solely to the $\mathrm{N}$ 1s $\rightarrow$ LUMO transition. The $\mathrm{N} 1 \mathrm{~s} \rightarrow$ LUMO+1 transition appears as a shoulder at $\sim 402.5 \mathrm{eV}$ and is also reproducible but underestimated in the calculated spectrum. The bonding character of these orbitals is similar to the corresponding orbitals in urea and thiourea with predominately $\pi^{*}$ character for the LUMO and $\sigma^{*}$ character for the LUMO+1. The next two transitions related to the experimental peak at $\sim 404 \mathrm{eV}$ contain both the $L U M O+2$ and the LUMO+3. The corresponding orbitals that contribute to these transitions are shown in Figure 6.

The main feature at $394.85 \mathrm{eV}$ in the experimental XE spectrum (Figure 4 c) ) contains two transitions. The dominant one is the HOMO-1 $\rightarrow \mathrm{N}$ 1s transition. The $\mathrm{N}$ contribution to this orbital is $\sim 50 \%$, much larger than the $5 \%$ contribution of the $\mathrm{N}$ site for the HOMO. Consequently the energetically higher $\mathrm{HOMO} \rightarrow \mathrm{N}$ 1s transition is almost vanishing in the XE spectrum. The calculated HOMO-2 is located at $392.84 \mathrm{eV}$. Here the $\mathrm{N}$ contribution is only $15 \%$ and no corresponding sharp peak can be found in the experimental spectrum. The experimental peak-to-peak value between the XE and XA main features of $\Delta E_{\text {exp }}^{p p}=6.10 \mathrm{eV}$ can be reproduced well by the calculated spectra with a peak-to-peak value of $\Delta E_{\text {calc }}^{p p}=5.87 \mathrm{eV}$, deviating less than $4 \%$ from the experiment. The calculated HOMO-LUMO gap of $\Delta E_{\text {calc }}^{H L}=5.45 \mathrm{eV}$, however, is $0.42 \mathrm{eV}$ smaller than the calculated peak-to-peak value because the XE peak is generated by the $\mathrm{HOMO}-1 \rightarrow \mathrm{N}$ 1s transition rather than the $\mathrm{HOMO} \rightarrow \mathrm{N}$ 1s. Taking the calculated energy-difference of the HOMO and the HOMO-1 into account the experimental HOMO-LUMO gap can be estimated to be $\Delta E_{\text {exp }}^{H L} \approx 5.70 \mathrm{eV}$ (cf. Table 1).

For dimethylurea, one hydrogen atom at each $\mathrm{N}$ site of urea is exchanged by a methyl group. Its geometry is depicted in Figure 1. The XE and XA spectra of dimethylurea (Figure $4 \mathrm{~d}$ )) are very similar to the spectra of the other molecules. At first glance, this is surprising since the local chemical surrounding of the $\mathrm{N}$ sites 
which is similar in thiourea, acetamide, and urea, containing one $\mathrm{C}-\mathrm{N}$ and two $\mathrm{N}-\mathrm{H}$ bonds, is different for dimethylurea, where the $\mathrm{N}$ site binds to one hydrogen and two carbon atoms. The XA spectrum of dimethylurea (Figure $4 \mathrm{~d}$ ), right part) is similar to the one of urea. The first peak at $402.1 \mathrm{eV}$ covers two transitions. The dominating $N$ 1s transition to the predominately $\pi^{*}$-like LUMO is closely followed by a transition to the $\sigma^{*}$-like LUMO+1.

The XE spectrum (Figure $4 \mathrm{~d}$ ), left part) is similar to the XE spectrum of urea as well, with its main feature located at $395.65 \mathrm{eV}$. The calculated line spectrum reveals that the peak is caused by transitions from the HOMO, HOMO-1, and HOMO-2 (cf. Figure $4 \mathrm{~d}$ ) and Figure 6). In contrast to the XE main peak of the three molecules discussed so far, the XE main peak of dimethylurea is actually dominated by the HOMO, due to a nitrogen contribution of more than $67 \%$ compared to a nitrogen contribution of $21 \%$ for the HOMO-1 and the HOMO-2, respectively. This characteristic is also reflected in the determination of the HOMO-LUMO gap. The calculated peak-topeak value is $\Delta E_{\text {calc }}^{p p}=6.15 \mathrm{eV}$, deviating from the calculated HOMO-LUMO gap of $\Delta E_{\text {calc }}^{H L}=5.90 \mathrm{eV}$ by only $0.25 \mathrm{eV}$. This is the smallest deviation among the molecules studied (cf. Table 1). With the experimental peak-to-peak value of $\Delta E_{\text {exp }}^{p p}=6.45 \mathrm{eV}$ the experimental HOMO-LUMO gap can be estimated to be $\Delta E_{\text {exp }}^{H L} \approx 6.2 \mathrm{eV}$, close to the theoretical value.

All four molecules discussed so far could be well reproduced by the calculations, including the relative changes in the energetic positions of the XA and XE main peaks among each other. All molecules showed strong similarities in the orbital structure of the high occupied and low unoccupied MOs. The character of the LUMO of all molecules could be identified as $\pi^{*}$ except for the N-H bonds, which exhibit a more $\pi$-like character, while the LUMO+1 had mostly $\sigma^{*}$ character. For the HOMO, the HOMO-1, and the HOMO-2 of each molecule the electron density was localized at the outer atoms, while the central $C$ atom had only a minor contribution in all cases. Nevertheless, comparing the peak-topeak values and the HOMO-LUMO gaps revealed that these two values can deviate significantly for each molecule. Hereby the emission spectrum plays a crucial role due to its sensitivity to the contribution of the specific atomic sites of the molecule to the MOs. However, by the use of calculations these contributions can be identified and therefore allow for an estimation of the actual HOMO-LUMO gap from the experimental XA and XE spectra. It has to be stressed here that the element under investigation (here $\mathrm{N}$ ) determines the deviation of the XE-peak-to-XA-peak value to the HOMO-LUMO gap.

To conclude the section about thiourea, acetamide, and dimethylurea, the RIXS spectra are discussed. For each of these three molecules in Figure 7 the experimental and calculated XES (top of each panel) and the RIXS spectra (bottom of each panel) are shown. The calculations of the latter ones were approximated by the XES calculations of the corresponding anions as described for urea. The general shift to lower emission energies in the RIXS experimental spectra is reproducible in the theoretical spectra for all three molecules, although the absolute value of the shift is slightly underestimated.

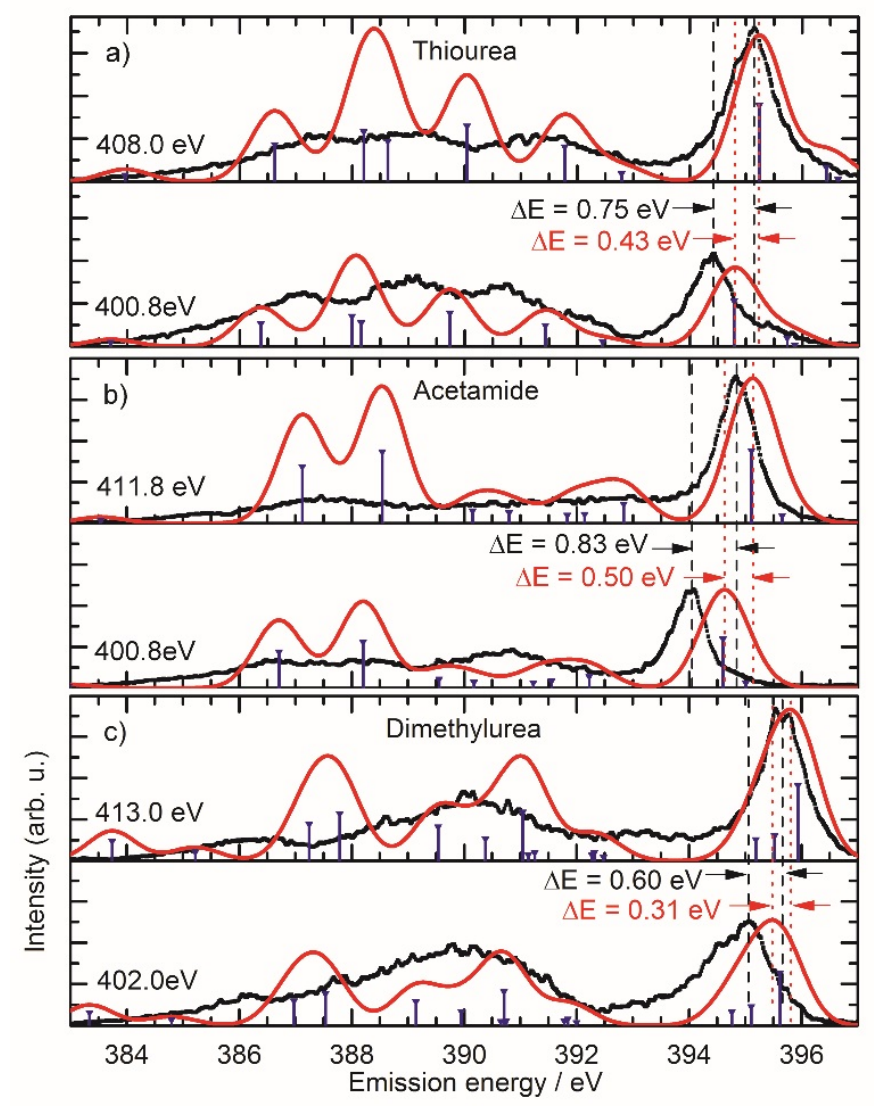

Figure 7. Experimental (black) and calculated (red) emission spectra for a) thiourea, b) acetamide, and c) dimethylurea. The top part of each panel shows the XE spectrum for the ionized molecule, and the bottom part of each panel the RIXS spectra at an excitation energy corresponding to the $\mathrm{N} 1 \mathrm{~s} \rightarrow$ LUMO transition. The blue bars indicate the calculated transition moments.

It shows for urea in Figure 5 that the main feature in the RIXS spectrum exhibits a shoulder leading to a broadening of the main peak by $\sim 45 \%$. Thiourea and acetamide, however, show a different behavior. The main peaks of thiourea and acetamide show no shoulder and have a very similar width for the ionized molecule (Figure 7 a) \& b), bottom) and for the excited molecule (Figure 7 a) \& b), top), i.e. $\sim 0.90 \mathrm{eV}$ for thiourea and $\sim 0.80 \mathrm{eV}$ for acetamide respectively. On the other hand dimethylurea exhibits a clear shoulder near the main emission feature, like urea, for the excited neutral molecule. Compared to the ionized molecule with a main peak of $\sim 1.00 \mathrm{eV}$ FWHM (Figure $7 \mathrm{c}$ ), top), the peak width for the excited neutral molecule is increased by $35 \%$ to a value of $\sim 1.35 \mathrm{eV}$ FWHM.

An explanation for this shoulder has been given in the aforementioned study of Weinhardt et al. ${ }^{[26]}$ on an aqueous solution of ammonia. The authors relate the low energy shoulder to an energy loss of the emitted photon to a proton during an ultrafast dissociation process, i.e. a proton transfer to the solvent. Assuming this explanation is correct, a new question arises: Why is this process occurring in urea and dimethylurea but not observable in thiourea and acetamide? An answer can be found in the structure of the molecular orbitals. For thiourea and acetamide a core electron excited with an energy close to the $\mathrm{N}$ 
absorption edge can only occupy the LUMO (cf. Figure 4 b) \& c) and Figure 6). For urea and dimethylurea, in contrast, the core electron can also occupy the LUMO+1, since it is energetically close to the LUMO. While the LUMO has in all cases $\pi$-like character concerning the $\mathrm{N}-\mathrm{H}$ bonds, the LUMO+1 has $\sigma^{*}$ character along these bonds. The excitation of an $\mathrm{N}$ 1s electron to the LUMO+1, therefore, might trigger a dissociation process. A straightforward way for a detailed study of this effect, however, would be the use of molecular dynamics calculations and experiments with deuterated molecules.

\section{II.3 Biuret}

The biuret molecule is special among the urea derivatives as it is the only one whose calculated absorption spectrum deviates significantly from the experimental one (Figure $4 \mathrm{e}$ ), blue line). Instead of the experimentally found single transition two transitions (marked with $\pi^{*}{ }_{1}$ and $\pi^{*}{ }_{2}$ ) appear in the calculation, deviating from the single experimental peak at $401.30 \mathrm{eV}$ approximately by $\pm 0.9 \mathrm{eV}$ (blue bars). As XA spectra are sensitive to the chemical environment the explanation of this incoherence is sought in the molecular structure. Biuret can be understood as two condensed urea molecules, i.e. it is composed of two carboxamide groups united by an $\mathrm{N}$ atom (Figure 1) which is chemically different from the $\mathrm{N}$ in the carboxamide groups. The calculated transitions from this atom appear at different energies and do not affect the results of the following considerations significantly, so that for the sake of clarity the central $\mathrm{N}$ is excluded from the following discussion.

Both carboxamide groups are identical fragments that would, if ideally separated, have their own set of localized orbitals at degenerate energies. However, in close vicinity as in the biuret molecule with only the central $\mathrm{N}$ atom between the fragments, orbital energies accordingly will split as for example has been discussed for X-ray absorption spectra of linear polyenes that display an $n$-fold splitting in the presence of $n$ conjugated double bonds forming $n \pi^{*}$ orbitals. ${ }^{[20]}$

For the "planar" biuret molecule, i.e. the two carboxamide planes are oriented in an angle of $\omega=180^{\circ}$ with respect to each other, a $\pi^{*}$ orbital splitting of $1.77 \mathrm{eV}$ is calculated (Figure 8, upper left side). Both molecular orbitals are delocalized over the whole molecule with one and two nodes, respectively, between the two $\pi^{\star}$ sub-systems.

In contrast, in the experimental spectrum no peak splitting is observed, indicating a less- or even un-coupled, localized $\pi^{*}$ system. The coupling, however, could be lifted through rotation of the fragments against each other as has been, e.g., analyzed before in the context of $\sigma$ electron delocalization in permethylated oligosilanes. ${ }^{[29]}$ There, it was shown by a theoretical rationalization that occupied and unoccupied orbitals with $\sigma$ bond character and hence $\sigma \sigma^{*}$ excitations depend strongly on the dihedral angles along the bonds separating two identical polysilane fragments. Two aspects from that piece of research find their counterparts here: rotation effects and orbital splitting.

To find a biuret configuration with smaller $\pi^{*}$ orbital splitting DFT and TD-DFT calculations were performed on a "twisted" conformer, i.e. the two carboxamide groups were rotated against each other. The smallest splitting of the $\pi^{\star}$ orbitals was found for a conformer with one of the $\mathrm{O}-\mathrm{C}-\mathrm{N}-\mathrm{C}$ dihedral angles being constrained to $\omega=90^{\circ}$ where both fragment planes are perpendicular to each other (Figure 8, right panel). Here both $\pi^{*}$ fragment orbitals are localized on only one of the carboxamide groups and the energy splitting decreases to $1.10 \mathrm{eV}$.

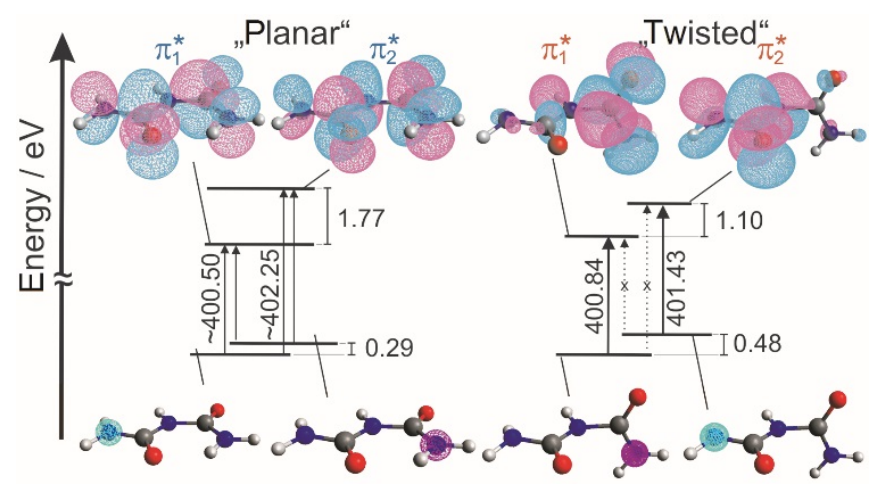

Figure 8. Energy positions of the $\mathrm{N} 1 \mathrm{~s}$ and $\pi^{*}$ orbitals of biuret in "planar" and "twisted" configuration. The planar conformer exhibits four transitions leading to two major contributions within the absorption spectrum at the indicated energies. For the twisted conformer two transitions are suppressed and the splitting is significantly reduced.

\section{Conclusions}

In the present study a systematic experimental and theoretical analysis of urea, thiourea, acetamide, dimethylurea, and biuret was conducted on the $\mathrm{N}$ K-edge by soft X-ray emission and absorption spectroscopy. The combination of experiment with DFT and TD-DFT calculations allows to connect the spectral features to the involved electronic transitions and corresponding electronic orbitals. Although the experimental spectra of the five investigated molecules appear to have similar shapes, the orbital contributions to individual spectral features differ significantly. This strongly affects the determination of the HOMO-LUMO gap from the experimental spectra. It was shown that the peak-to-peak values obtained from the XE and XA spectra overestimate the HOMO-LUMO gap in all cases. The deviation of the peak-to-peak value from the HOMO-LUMO gap, however, can be estimated by comparing the calculated transition energies to the peak positions in the calculated broadened spectra. By taking this deviation into account for the experimental spectra reliable values of the HOMO-LUMO gap were obtained (cf. Table 1). The HOMOLUMO gap of urea is determined to be $\Delta E_{\text {exp }}^{H L} \approx 6.75 \mathrm{eV}$, which is considerably larger than the value of $6.2 \mathrm{eV}$ for crystalline urea. The urea derivatives all exhibit smaller values, with thiourea having the lowest $\Delta E_{\text {exp }}^{H L} \approx 4.30 \mathrm{eV}$.

Further in this study the RIXS spectra were investigated. The observed energetic shifts could qualitatively be reproduced by calculating the X-ray emission spectra for the molecules in an anionic state, and the main peak broadening appearing in the RIXS spectra of urea and dimethylurea is assigned to an ultrafast dissociation process of a proton, which is triggered by the occupation of the $\sigma^{*}$-like LUMO+1 orbital.

In addition, this systematic study shows that a universal energy shift (here $20.2 \mathrm{eV}$ ) of all calculated spectra for all five molecules 
(except the absorption spectrum for biuret in a "planar" geometry) is sufficient to match their respective experimental spectra. For the exceptional case of biuret, it is found that a modification of the molecular geometry can drastically reduce this mismatch, suggesting a twisted molecular structure, instead of the planar structure obtained by the single molecule calculations, present in the solvated condition.

\section{Experimental Section}

In this study commercially available samples, bought from SigmaAldrich were used in a purity $>99 \%$ for urea, thiourea, and acetamide, $>97 \%$ for biuret, and $>95 \%$ for dimethylurea. The samples were dissolved in $\mathrm{H}_{2} \mathrm{O}$ to a concentration of $1 \mathrm{M}$ for urea, acetamide, thiourea, and dimethylurea. For biuret the concentration was $0.2 \mathrm{M}$ due to its reduced solubility. The liquid microjet technique ensures a continuous renewal of the sample, therefore experimental artifacts caused by sample damage due to the X-ray illumination can be excluded. ${ }^{[1,3]}$ The element selectivity of the X-ray technique ensures that the measured spectra in this study stems only from the element under investigation and is not superimposed by signals from the solvent.

The room temperature experiments were performed using the experimental endstation LiXEdrom at the synchrotron facility BESSY II in Berlin, Germany, at the undulator beamline U49/2 $P G M-1^{[30]}$. The energy resolution of the beamline was set to $E / \Delta E=1600$, leading to an energy resolution of $\sim 250 \mathrm{meV}$ in the presented XA spectra. The detector, used to measure the emitted photons, comprises a high resolution grating with 1200 lines $/ \mathrm{mm}$ optimized for an energy range of $200-500 \mathrm{eV}$ and a CCD camera in combination with a multichannel plate and a fluorescence screen. The detector was calibrated to the incident photon energy using the elastic scattering line (incident photon energy = emitted photon energy) at the $\mathrm{N} \mathrm{K}$-edge of a solid $\mathrm{BN}$ sample. Subsequently, the elastic features of the investigated molecules were checked to exclude energy shifts. Thereby, the absolute energy positions of the main features in the XE and RIXS spectra of the individual molecules could be determined with an accuracy better than $100 \mathrm{meV}$ and an energy resolution of $\sim 300 \mathrm{meV}$.

The principle of the three techniques used in this study is based on the excitation of a core electron and the subsequent relaxation of valence electrons to refill the core hole (Figure 9). In the XA process the incident photon energy is tuned across the absorption edge, exciting the $\mathrm{N} 1 \mathrm{~s}$ electron into empty molecular orbitals, including the orbitals above the ionization potential (IP) to ionize the molecule. The XA measurements in this study were performed in partial fluorescence yield (PFY) mode using the MCP detector. The detection range covers photon energies from approximately $375.0 \mathrm{eV}$ to $410.0 \mathrm{eV}$.

In the other two processes the emitted photons created during the relaxation are detected in an energy-resolved fashion. For high incident photon energies, i.e. for the ionized molecule, the technique is called XES. For an incident photon energy below the ionization potential the electron is excited to an unoccupied molecular orbital. The additional electron at the LUMO or above acts on the overall electronic configuration causing a rearrangement of the electronic structure, which can be detected in the energy-resolved emission spectrum. This is the so-called RIXS process. ${ }^{[31]}$

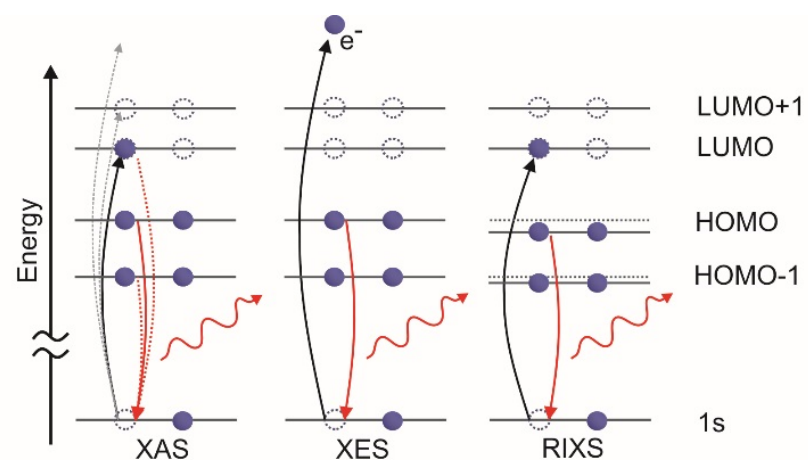

Figure 9. Scheme of the XAS, XES, and RIXS processes.

\section{Computational Section}

Geometries were optimized by density functional theory (DFT) and the spectra were calculated using DFT and time-dependent DFT (TD-DFT) with the ORCA program package version 3.0.3 ${ }^{[32]}$ with BP86-D3/def2-TZVP ${ }^{[33-39]}$ functional and basis set under tight convergence criteria. The functional was chosen after comparison of calculations with the functionals TPSSh, ${ }^{[40]}$ TPSS0, ${ }^{[41]}$ and $B 3 L P^{[42]}$ on acetamide that gave similar geometries but lead to an underestimation of the splitting among the two lowest-energy absorption peaks in comparison to experimental findings. ${ }^{[43]}$ Water as solvent was taken into account via the conductor-like screening model (COSMO $)^{[44]}$ for geometry optimizations as well as for the final single point calculations. In this study the RI approximation ${ }^{[45-48]}$ was used together with consistent auxiliary basis sets. ${ }^{[49]}$ The general procedure started with geometry optimizations followed by frequency calculations to assure the stationary geometry to be a minimum. The geometrically optimized structures of the five investigated molecules urea, acetamide, thiourea, biuret, and dimethylurea in this paper are shown in Figure 1. In the case of biuret geometry optimization for the twisted conformer was carried out with constraints regarding one of the O-C-N-C dihedral angles. Orbital energies and HOMOLUMO gaps were obtained from single point DFT calculations in the optimized geometries. N K-edge absorption spectra were calculated with TD-DFT, emission spectra with a DFT calculation on the neutral molecule (rather than the core-ionized cation) in $X E S^{[50]}$ and on the anion with the additional electron in the LUMO, corresponding to the $\mathrm{N}$ 1s $\rightarrow$ LUMO transition, for RIXS. In this DFT emission spectroscopy approach transition-bar-positions are deduced directly from orbital - rather than state - energy differences and relative peak intensities for the transitions from any final state to the initial core-hole state are given through the respective transition dipole moment expectation values based on the DFT orbitals mentioned above. ${ }^{[50]}$ All spectra obtained with BP86-D3/def2-TZVP were shifted by a constant value of $20.2 \mathrm{eV}$ to match the experimental positions as it is typically done to account for the lower accuracy of DFT core orbitals compared to 
valence orbitals which leads to an overestimation of core orbital energies. ${ }^{[51]}$ The orbital plotting was performed using the program Avogadro. ${ }^{[52,53]}$

\section{Acknowledgements}

K.A. acknowledges the financial support of the Einstein Foundation Berlin for a postdoctoral scholarship and A.B. acknowledges the financial support of the Volkswagen Foundation for her position within the Freigeist fellowship.

Keywords: Ureas $\cdot \mathrm{X}$-ray spectroscopy $\bullet$ liquids $\bullet$ frontier orbitals $\cdot$ density functional theory

[1] H. Siegbahn, K. Siegbahn, J. Electron Spectrosc. Relat. Phenom., 1973, 2, 319-325.

[2] M. Faubel, B. Steiner, J. P. Toennies, J. Chem. Phys., 1997, 106, 90139031.

[3] K. M. Lange, E. F. Aziz, Chem. Soc. Rev., 2013, 42, 6840-6859.

[4] B. X. Yang, J. Kirz, Phys. Rev. B, 1987, 36, 1361(R).

[5] B. Winter, M. Faubel, Chem. Rev., 2006, 106, 1176-1211.

[6] S. Schreck, G. Gavrila, C. Weniger, P. Wernet, Rev. Sci. Instrum., 2011, 82, 103101.

[7] R. Dovesi, M. Causa, R. Orlando, C. Roetti, V. R. Saunders, J. Chem. Phys., 1990, 92, p. 7402.

[8] A. Gobbi, G. Frenking, J. Am. Chem. Soc., 1993, 115, 2362-2372.

[9] R. V. Belosludov, L. Z.-Q., Y. Kawazoe, Mol. Eng., 1999, 8, 105-119.

[10] R. W. Gora, W. Bartkowiak, S. Rozsak, J. Chem. Phys., 2002, 117, 1031 1039.

[11] J. Grdadolnik, Y. Maréchal, J. Mol. Struct., 2002, 615, 177-189.

[12] F. Sokolic, A. Idrissi, A. Perera, J. Mol. Liq., 2002, 101, 81-87.

[13] Y. M. Jung, B. Czarnik-Matusewicz, S. B. Kim, J. Phys. Chem. B, 2004, 108, 13008-13014

[14] A. Idrissi, Spectrochim. Acta, Part A, 2005, 61, 1-17.

[15] Y. Hayashi, Y. Katsumoto, S. Omori, N. Kishii, A. Yasuda, J. Phys. Chem. B, 2007, 111, 1076-1080.

[16] D. E. Manolopoulos, J. C. May, S. E. Down, Chem. Phys. Lett., 1991, 181, 105-111.

[17] M. M. Lynam, M. Kuty, J. Damborsky, J. Koca, P. Adriaens, Environ. Toxicol. Chem., 1998, 17, 988-997.

[18] J.-i. Aihara, J. Phys. Chem. A, 1999, 103, 7487-7495.

[19] J.-i. Aihara, Phys. Chem. Chem. Phys., 2000, 2, 3121-3125.

[20] J. Stöhr, NEXAFS spectroscopy, Springer-Verlag, Berlin, 1992.

[21] F. A. Asmuruf, N. A. Besley, Chem. Phys. Lett., 2008, 463, p. 267.

[22] R. Adams, H. H. M. Balyuzi, R. E. Burge, J. Appl. Cryst., 1977, 10, 256261.
[23] X. Hoccard, G. Turrell, J. Chem. Phys., 1993, 99, p. 8498.

[24] J. Jaklevic, J. A. Kirby, K. M. P., A. S. Robertson, G. S. Brown, E. P., Solid State Commun., 1977, 23, 679-682.

[25] C. Cassidy, J. M. Halbout, W. Donaldson, C. Tang, Opt. Commun., 1979, 29, no. 2, pp. 243-246.

[26] L. Weinhardt, M. Weigand, O. Fuchs, M. Bär, M. Blum, J. D. Denlinger, W. Yang, E. Umbach, C. Heske, Phys. Rev. B, 2011, 84, 104202.

[27] E. F. Aziz, N. Ottosson, M. Faubel, I. V. Hertel, B. Winter, Nature, 2008 , 455, 89-91.

[28] B. Ravi, A. Jegatheesam, B. Neelakandaprasad, C. Sadeeshkumar, G. Rajarajan, Rasayan J. Chem., 2014, 7, 387-294.

[29] A. Bande, J. Michl, Chem. Eur. J., 2009, 15, 8504-8517.

[30] K. J. S. Sawhney, F. Senf, W. Gudat, Nucl. Instrum. Methods Phys. Res., Sect. A, 2001, 467-468, 466-469.

[31] J. P. A. Luuk, M. van Veenendaal, T. P. Devereaux, J. P. Hill, J. van den Brink, Rev. Mod. Phys., 2011, 83, 705-767.

[32] F. Neese, WIREs Comput. Mol. Sci., 2012, 2, 73-78.

[33] A. D. Becke, Phys. Rev. A, 1988, 38, 3098-3100.

[34] J. P. Perdew, Phys. Rev. B, 1986, 33, 8822-8824.

[35] J. P. Perdew, Phys. Rev. B, 1986, 34, p. 7406.

[36] S. Grimme, J. Antony, S. Ehrlich, H. Krieg, J. Chem. Phys., 2010, 132, 154104.

[37] S. Grimme, S. Ehrlich, L. Goerigk, J. Comp. Chem., 2011, 32, 1456-1465

[38] A. Schäfer, H. Horn, R. Ahlrichs, J. Chem. Phys., 1992, 97, 2571-2577.

[39] F. Weigend, R. Ahlrichs, Phys. Chem. Chem. Phys., 2005, 7, 3297-3305.

[40] V. N. Staroverov, G. E. Scuseria, J. Tao, J. P. Perdew, J. Chem. Phys., 2003, 119, $12129-12137$.

[41] S. Grimme, J. Phys. Chem. A, 2005, 109, p. 3067-3077.

[42] A. D. Becke, J. Chem. Phys., 1993, 98, 5648-5652.

[43] M. Kitano and K. Kuchitsu, Bull. Chem. Soc. Jpn., 1973, 46, 3048-3051.

[44] S. Sinnecker, R. Arivazhagan, A. Klamt, M. Diedenhofen, F. Neese, J. Phys. Chem. A, 2006, 110, 2235-2245.

[45] E. J. Baerends, D. E. Ellis, P. Ros, Chem. Phys., 1973, 2, 41-51.

[46] J. L. Whitten, J. Chem. Phys., 1973, 58, 4496-4501.

[47] B. I. Dunlap, J. W. D. Connolly, J. R. Sabin, J. Chem. Phys., 1979, 71, p. 3396-3402.

[48] C. Van Alsenoy, J. Comput. Chem., 1988, 9, 620-626.

[49] F. Weigend, M. Häser, Theor. Chem. Acc., 1997, 97, 331-340.

[50] N. Lee, T. Petrenko, U. Bergmann, F. Neese, S. DeBeer, J. Am. Chem. Soc., , 2010, 132, 9715-9727.

[51] S. DeBeer George, T. Petrenko, F. Neese, J. Phys. Chem. A, 2008, 112, 12936-12943,

[52] Avogadro: an open-source molecular builder and visualization tool. Version 1.1.1 http://avogadro.openmolecules.net/.

[53] M. Hanwell, D. Curtis, D. Lonie, T. Vandermeersch, E. Zurek, G. Hutchison, J. Cheminform., 2012, 4:17. 